\title{
Dynamics of polybutadienes with different microstructures. 2. Dielectric response and comparisons with rheological behavior
}

\author{
Reiner Zorna) \\ Forschungszentrum Jülich, IFF, D-52425 Jülich, Germany \\ Frederick I. Mopsik and Gregory B. McKenna ${ }^{\text {b) }}$ \\ National Institute of Standards and Technology, Gaithersburg, Maryland 20899 \\ Lutz Willner and Dieter Richter \\ Forschungszentrum Jülich, IFF, D-52425 Jülich, Germany
}

(Received 19 February 1997; accepted 30 May 1997)

\begin{abstract}
A series of 1,2-1,4-polybutadienes with varying 1,2 (vinyl) content was investigated using time-domain dielectric spectroscopy. The time range was $10^{-5}-300 \mathrm{~s}$, which can be converted by Laplace transform to a frequency range of $10^{-3}-6000 \mathrm{~Hz}$. The samples were the same as those used in a previous rheological study from these laboratories. Therefore, a direct comparison of dielectric and mechanical responses was possible. Within experimental uncertainty, the $\alpha$ relaxation observed by both methods shows the same temperature dependence but there is an offset between the characteristic times of both methods, which increases with increasing vinyl content. This result can be qualitatively understood from the difference of the size of the dipolar groups, viz. cis and vinyl monomeric units, in the context of the DiMarzio-Bishop model. In addition, the question of time-temperature superposition was studied using the dielectric data. In the cases of a vinyl content $\geqslant 0.53$ no deviations from time-temperature superposition were detected. Only for the sample with the lowest vinyl content (0.07) does the attempt to construct a master curve from the dielectric loss data fail. In this case a fit with a combination of a Havriliak-Negami and a Cole-Cole functions suggests that this deviation from time-temperature superposition is an intrinsic feature of the $\alpha$ relaxation rather than an effect of its merging with the $\beta$ relaxation. The absence of indications for such a deviation in the rheological study can be explained by the smaller frequency range of the latter. This stresses the necessity of a large dynamic range in experiments aimed at the examination of the time-temperature superposition principle. (C) 1997 American Institute of Physics.
\end{abstract} [S0021-9606(97)50833-6]

\section{INTRODUCTION}

Dielectric measurements on glass-forming systems (especially polymers) have a long tradition and have revealed several general phenomena.

First, an intensive loss peak is detected which shifts strongly with temperature. Because this is often the peak occurring at lowest frequency (or highest temperature if the frequency is held constant) it is usually referred to as the $\alpha$ peak. The relaxation associated with this peak is generally reported to show the same temperature dependence as the mechanical relaxation. ${ }^{1}$ It is normally associated with the same molecular-level events that give rise to mechanical relaxations on a large length scale. The peak shape, which is not a simple Debye function, is often described phenomenologically by means of the Havriliak-Negami function ${ }^{2}$ in the frequency domain or the Kohlrausch-Williams-Watts law ${ }^{3,4}$ in the time domain. Both representations give asymmetric peak shapes of the loss part represented on a logarithmic frequency scale, but have different asymptotic behaviors at low frequencies in the frequency domain.

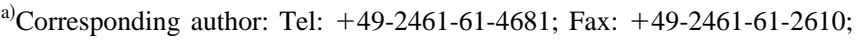
Electronic-mail: R.Zorn@kfa-juelich.de

${ }^{b)}$ Corresponding author: Tel: 301-975-6752; fax: 301-977-2018; Electronicmail: greg@micf.nist.gov
}

Second, a smaller peak which shows a weaker temperature dependence is observed at higher frequency. This so-called $\beta$ peak exhibits a rather symmetric shape and is described phenomenologically by, e.g., the Cole-Cole ${ }^{5}$ function or a log-normal distribution. ${ }^{6}$ Both reflect the fact that this loss peak is symmetric on a logarithmic frequency scale. The origins of this $\beta$ peak are still unclear. Sometimes it has been related to material-specific motions (especially of sidegroups in polymers) ${ }^{1}$ but due to its ubiquity other authors have concluded that the $\beta$ relaxation is a more general phenomenon related to the glass transition. ${ }^{7}$

Because of the different temperature dependencies of the peak positions of the $\alpha$ and $\beta$ relaxations there should be a temperature region where the loss peaks merge. This region is still a subject of controversy. ${ }^{8}$ One reason for the conflicting views is that when both peaks begin to overlap the procedure for decomposition into $\alpha$ and $\beta$ contributions influences the results strongly. ${ }^{9}$

In a previous paper we presented results from an investigation of the dynamic mechanical response of a series of polybutadienes having different microstructures. ${ }^{10}$ Here we extend that investigation using time-domain dielectric spectroscopy measurements that cover 7.5 decades in time (transformed to 6.7 in frequency) rather than the 4 decades used in the mechanical measurements. The larger dynamical range is 
TABLE I. Molecular weights, microstructures, and glass transition temperatures of anionically polymerized polybutadienes used in this investigation.

\begin{tabular}{ccccccc}
\hline \hline Sample & $M_{w}{ }^{\mathrm{a}} / 10^{3} \mathrm{~g} \mathrm{~mol}^{-1}$ & $\begin{array}{c}1,2^{\mathrm{b}} \\
\text { fraction }\end{array}$ & $\begin{array}{c}1,4-\text { trans }^{\mathrm{b}} \\
\text { fraction }\end{array}$ & $\begin{array}{c}1,4-\text { cis }^{\mathrm{b}} \\
\text { fraction }\end{array}$ & $T_{g, \text { DSC }}\left({ }^{\circ} \mathrm{C}\right)$ & $T_{g, \text {,heo }}\left({ }^{\circ} \mathrm{C}\right)$ \\
\hline PB7 & 19 & $0.07(0.07)$ & 0.52 & 0.41 & -95 & -98.0 \\
PB53 & 18 & $0.52(0.53)$ & 0.29 & 0.19 & -57 & -60.4 \\
PB70 & 16 & $0.68(0.70)$ & 0.20 & 0.12 & -35 & -41.9 \\
PB95 & 19 & $0.95(0.95)$ & $\ldots c^{\mathrm{c}}$ & $\cdots$ & -1 & -4.1 \\
\hline \hline
\end{tabular}

${ }^{\mathrm{a} B y}$ light scattering in cyclohexane.

${ }^{\mathrm{b}} \mathrm{By}{ }^{13} \mathrm{C}$ NMR, the values in parentheses are by ${ }^{1} \mathrm{H} \mathrm{NMR}$.

${ }^{c} 1,4$-cis +1,4-trans: 0.05 .

a general advantage of dielectric spectroscopy. In this paper we show that the dynamical range of the measurements may significantly affect data interpretation, particularly the apparent validity of time-temperature superposition.

In this investigation the same 1,2-1,4-polybutadienes were used as in the previous rheological study. ${ }^{10}$ These polymers were synthesized by anionic polymerization. By changing the conditions of the synthesis (e.g., the solvent or temperature) the 1,2 (vinyl) content was controlled over the range 0.07-0.95. Hence, these polymers offer the opportunity for studying the influence of an increasing number of short, rigid side groups on the otherwise unchanged polymer. A well-known consequence of this modification is a strong increase in the glass-transition temperature $T_{g}$ with increasing vinyl content.

Our previous study of the rheological response of the materials ${ }^{10}$ showed a change of the temperature dependence of the shift factor, an increase in fragility, ${ }^{11,12}$ and a change of the stretching exponent of the rheological $\alpha$ relaxation with increasing vinyl content. Time-temperature superposition was shown to be successful in the description of both the glassy dispersion and the terminal dispersion. The large dynamical range covered by the dielectric data allows the question of time-temperature superposition to be reexamined.

\section{EXPERIMENTAL METHODS}

\section{A. Materials}

The four polybutadienes used in this investigation were prepared by anionic polymerization using high vacuum and break-seal techniques, generally following the procedures outlined by Morton and Fetters. ${ }^{13}$ A detailed description of the synthesis and characterization of these samples can be found elsewhere. ${ }^{10}$ Table I depicts the molecular weights and microstructures of these polybutadienes. Also shown are the glass-transition temperatures $T_{g}$ reported previously from both differential scanning calorimetry (DSC) and rheological measurements at an arbitrary relaxation time of $\tau_{M}=10 \mathrm{~s}$.

In order to avoid degradation of the samples during their storage $\approx 0.1 \%$ of stabilizer (2,6-di-tert-butyl-4-methylphenol) was added. The first dielectric measurements showed that either this stabilizer or residual Li cations from the polymerization initiator caused a considerable amount of conductivity. Therefore all samples were dissolved in tetrahydrofurane and precipitated in a methanol/water $/ \mathrm{HCl}$ mixture five times immediately before the experiment. After this procedure only minor traces of conductivity could be observed.

\section{B. Dielectric measurements (time-domain spectroscopy)}

The dielectric measurements were performed using a time-domain spectrometer designed and built by Mopsik. ${ }^{14}$ This spectrometer allows high accuracy $(0.1 \%$ of the total dispersion $\Delta \epsilon$ ) and high sensitivity $\left(\tan \delta=10^{-5}\right)$ over a large time range.

The raw data are taken by applying a voltage step $\left(E^{0}\right.$ $=100 \mathrm{~V})$ to the sample and measuring the resulting charge $Q(t)$ on the sample capacitor over a time range from $10^{-5}$ to $300 \mathrm{~s}$. (If the leakage current of the sample allows, the long time limit can be increased.) Capacity data $C(t)=Q(t) / E^{0}$ were aquired via an analog-digital converter on a personal computer during the time of the experimental observation.

These data were then converted to the frequency domain by a Laplace transform:

$$
C^{*}(\omega)=\lim _{v \rightarrow 0+} \int_{0}^{\infty} \exp (-s t) \dot{C}(t) d t
$$

with $s=v+i \omega$.

The transform is executed by a technique developed to treat data that is logarithmically spaced over a large range. ${ }^{15} \mathrm{It}$ yields a frequency range of $10^{-3}-6 \times 10^{3} \mathrm{~Hz}$ in $C^{*}(\omega)$ $=C^{\prime}(\omega)-i C^{\prime \prime}(\omega)$.

The sample cell was constructed from two epoxy-resin printed circuit (PC) boards. Each board had a 25-mm-diam sample capacitor electrode etched into it. A guard ring was etched around one of these. The sample was placed between the circuit sides of the PC boards and a spacer foil was used to maintain a thickness of about $0.25 \mathrm{~mm}$. The resulting empty cell capacitance was $C_{0} \approx 19 \mathrm{pF}$. This latter value could not be determined with high precision because after closing the sample cell only its total thickness can be measured and the error after subtracting the thicknesses of the PC boards amounts to $0.05 \mathrm{~mm}$, i.e., approximately $20 \%$ relative error. Furthermore, a $30 \%$ reduction in the capacity values could be detected after some of the experiments (which lasted about one week for each sample). It is probable that the PC boards were deformed in loading the samples and relaxed due to sample flow during the experiments. There- 

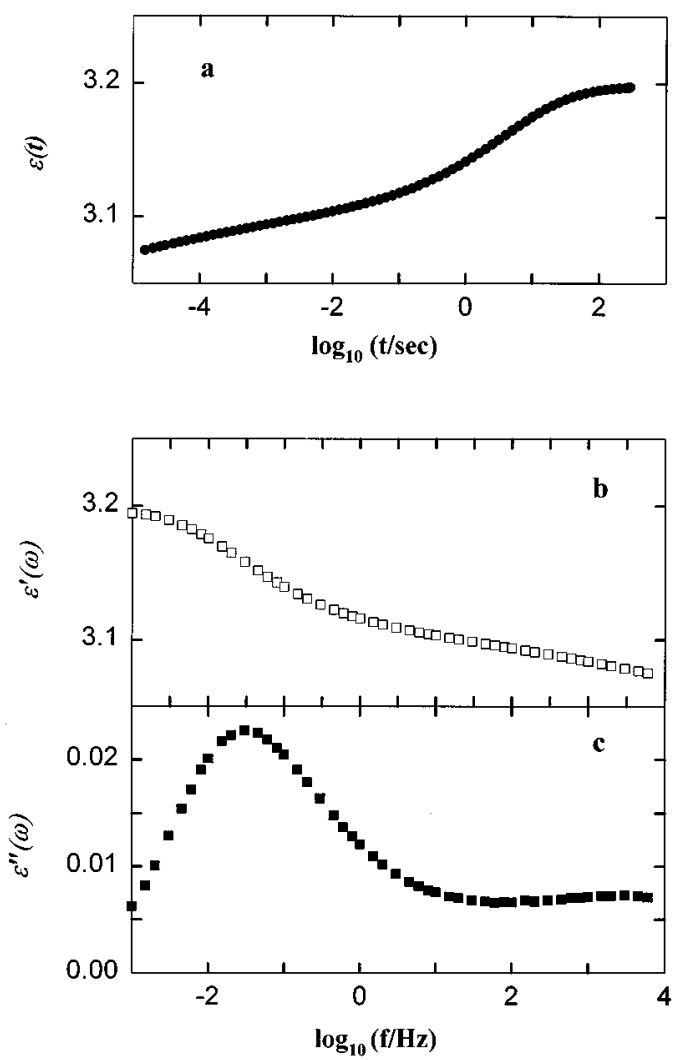

FIG. 1. (a) Time-domain spectroscopy data of PB 7 at $-97.7^{\circ} \mathrm{C}$. (b), (c) Real (storage) and imaginary (loss) part of the dielectric function obtained by transforming the data (a) into the frequency domain.

fore, the absolute value of $\epsilon^{*}(\omega)=\epsilon^{\prime}(\omega)-i \epsilon^{\prime \prime}(\omega)$ $=C^{*}(\omega) / C_{0}$ is affected with a large error ${ }^{16}$ but the value of $\tan \delta=\epsilon^{\prime \prime}(\omega) / \epsilon^{\prime}(\omega)$ is precise to the accuracy of the electronic circuit. It has to be noted that the small changes of the sample geometry were very slow compared to the measuring time, so the shape of $\epsilon^{*}(\omega)$ is not distorted by them.

Temperature was controlled using a liquid nitrogen cryostat that was constant to within $0.02 \mathrm{~K}$ range over the $-130-+50{ }^{\circ} \mathrm{C}$ used during our experiments. The temperature was measured by a Pt100 resistor which was calibrated against NIST standards. The error of the absolute temperatures can be estimated to be $\approx 0.5 \mathrm{~K}$.

\section{RESULTS}

Figure 1(a) shows a representative data set in the time domain from the PB 7 sample at $-97.7^{\circ} \mathrm{C}$. Figures 1(b) and 1 (c) show the real and imaginary parts of the complex dielectric function obtained by the transform described above. The imaginary part $\epsilon^{\prime \prime}(\omega)$ reveals two maxima: a sharper one at $f=\omega / 2 \pi=35 \mathrm{mHz}$ and a very broad one at $\approx 3 \mathrm{kHz}$. These features are only weakly visible visible in the time domain data and in the real part $\epsilon^{\prime}(\omega)$.

Figure 2 exhibits all data from this sample in a threedimensional plot of the loss tangent $\tan \delta$ versus the logarithm of the frequency and temperature. We draw the reader's attention to three features: (1) A slightly curved "crest"

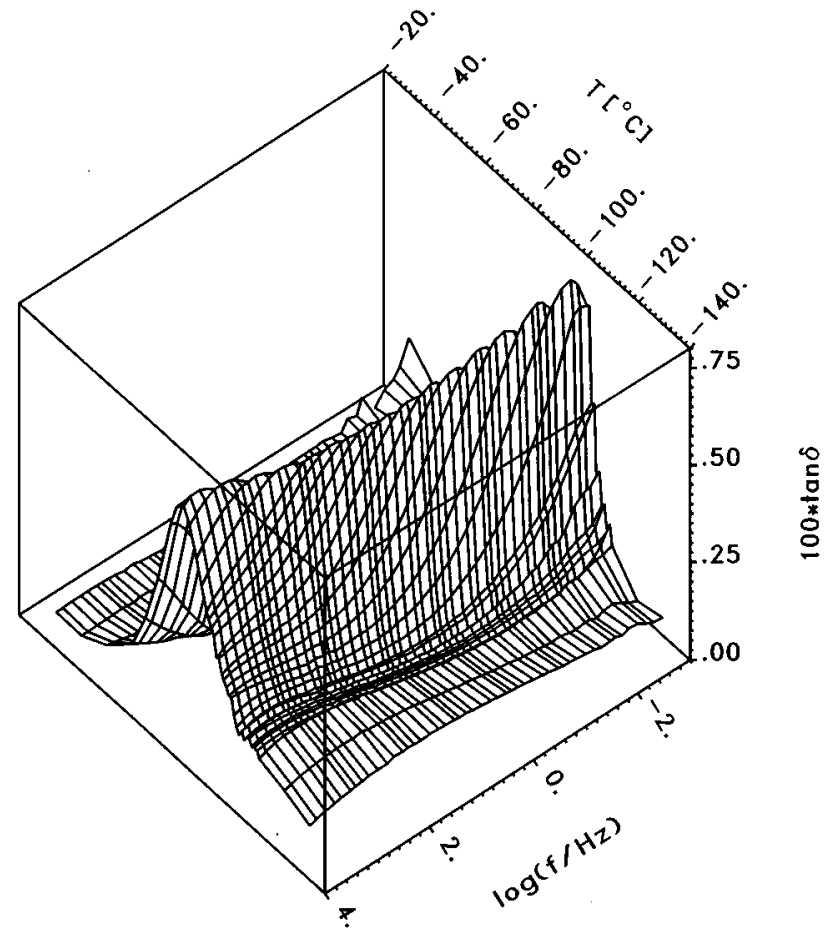

FIG. 2. Three-dimensional representation of the loss tangent $\tan \delta$ vs the logarithm of the frequency and temperature for sample PB 7. The figure comprises 36 spectra at temperatures between -129.6 and $-29.8^{\circ} \mathrm{C}$.

associated with a maximum that occurs in the $\tan \delta$ temperature plane. Its width is about $20 \mathrm{~K}$ and it occurs over the entire frequency range. This is the $\alpha$ relaxation. (2) A much less pronounced "mound" around $1 \mathrm{kHz}$ which is also present at the lowest temperatures. This is the $\beta$ relaxation. (3) An upturn in the corner of high temperature/low frequency. The last item is due to residual conductivity which is still present despite the purification of the sample.

\section{DISCUSSION}

\section{A. Analysis of the data in frequency space}

In order to quantify the different contributions to the dielectric function, we have chosen to describe the $\alpha$ relaxation by a Havriliak-Negami (HN) function ${ }^{2}$

$$
\epsilon^{*}(\omega)=\epsilon_{\infty}+\frac{\epsilon_{0}-\epsilon_{\infty}}{\left[1+\left(i \omega \tau_{\mathrm{HN}}\right)^{\alpha}\right]^{\gamma}}
$$

and the $\beta$ relaxation by a Cole-Cole function, ${ }^{5}$ which is the special case of Eq. (4.1) with $\gamma=1$. The sum of the imaginary parts was fitted to the $\epsilon^{\prime \prime}(\omega)$ data.

This combination did not fit the data adequately for temperatures where the $\alpha$ maximum was at frequencies $f_{\max }$ $>1 \mathrm{~Hz}$. In these cases the low frequency wings of the peaks seem to show an additional contribution in $\epsilon^{\prime \prime}(\omega)$. This contribution could not be ascribed to a residual conductivity in the sample. Therefore, we arrived at the conclusion that it is due to a low frequency relaxation in the PC boards used for the sample holder. The epoxy-fiberglass composite these boards are made from was recently studied by dielectric 


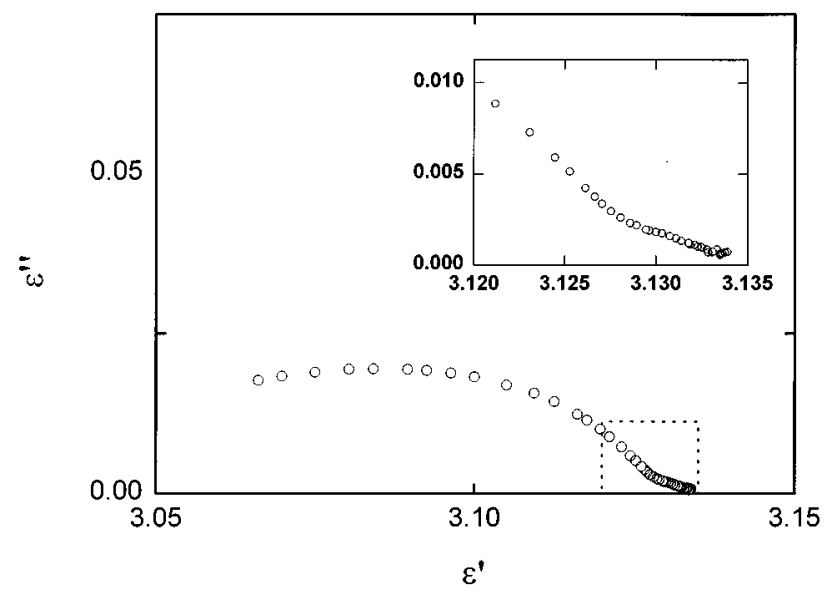

FIG. 3. Cole-Cole plot $\left(\epsilon^{\prime \prime}\right.$ vs $\left.\epsilon^{\prime}\right)$ of the dielectric function of PB 7 at $-82.3^{\circ} \mathrm{C}$. The filled dots indicate the frequencies $10^{3}, 10^{2}, \ldots, 10^{-3} \mathrm{~Hz}$ from left to right. The insert shows an enlargement of the low frequency lobe.

measurements, ${ }^{19}$ which revealed a low frequency relaxation arising mainly from the Maxwell-Wagner mechanism associated with the interfaces in the material. The frequency range affected by this artifact was subsequently ignored in the fits.

It is also possible to use a Kohlrausch-Williams-Watts $(\mathrm{KWW})^{3,4}$ function to fit the $\alpha$ relaxation. We chose not to do this sort of data analysis because of the asymptotic behavior of the polybutadiene data we obtained. The KWW function is given in the time domain by

$$
\Phi_{\mathrm{KWW}}(t)=\exp \left[-\left(t / \tau_{\mathrm{KWW}}\right)^{\beta}\right]
$$

and in the frequency domain its asymptotic behavior at low frequencies is given as

$$
\epsilon^{\prime}(\omega) \sim \omega^{0}, \quad \epsilon^{\prime \prime}(\omega) \sim \omega^{1} .
$$

For the Cole-Cole plot $\left(\epsilon^{\prime \prime}\right.$ vs $\left.\epsilon^{\prime}\right)$ of our polybutadiene data shown in Fig. 3 this would imply a right angle at the intersection of the arc and the axis on the right-hand (low frequency) side. Although this side is distorted by the effect of the epoxy PC boards mentioned above, the data obviously do not come into the axis at a $90^{\circ}$ angle, implying that the variation of $\epsilon^{\prime \prime}(\omega)$ with $\omega$ is weaker than proportional for low frequencies. The same result was found by Deegan and Nagel $^{20}$ in the case of 0.20 vinyl-fraction polybutadiene and was given as evidence that the Dixon-Nagel ${ }^{21}$ scaling relation does not hold for the case of polybutadiene.

Therefore the HN function, which has as a low frequency asymptote a power law dependence on frequency of the loss

$$
\epsilon^{\prime \prime}(\omega) \sim \omega^{\alpha}
$$

with $\alpha<1$, is a better descriptor of the data obtained here than is the KWW function. The corresponding angle in the Cole-Cole plot is $\arctan (\alpha \pi / 2)$ and can have any value between $0^{\circ}$ and $90^{\circ}$. Of course, a fit with the $\mathrm{HN}$ function is done at the expense of having an additional free exponent parameter. Also, the shape (and especially the width) of a

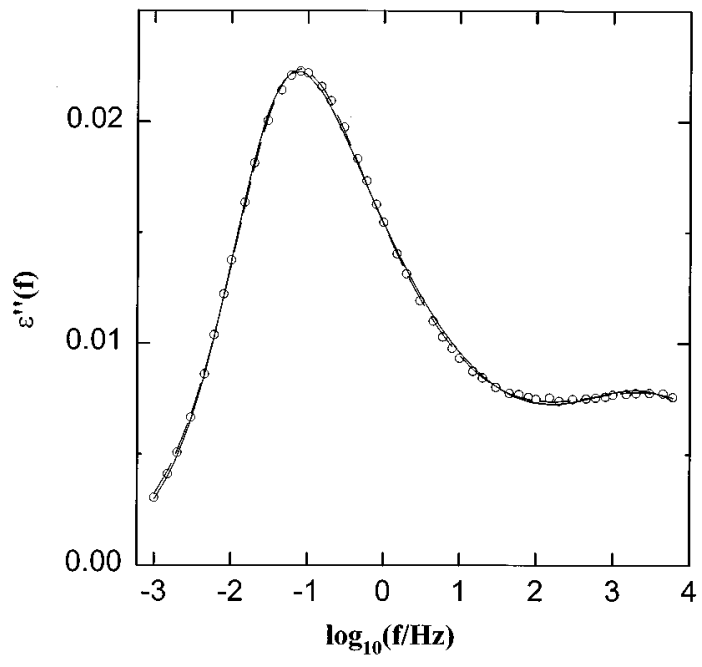

FIG. 4. Symbols: Loss part of the dielectric function $\epsilon^{\prime \prime}(\omega)$ for PB 7 at $-96.7^{\circ} \mathrm{C}$. Lines: Fits with a sum of the HN and CC functions, (---) with the HN parameter $\gamma=0.32$ fixed, (---) with HN parameter $\gamma$ fitted.

spectrum now depends on two parameters- $\alpha$ and $\gamma$-rather than just the $\beta$ in the KWW function. This fact may complicate the comparison of HN functions from different samples or different temperatures for the same sample. Whether one combination of exponent parameters results in a wider spectrum than another may depend on the definition of "width" for certain parameter combinations.

In the case of the data we report here, it was possible to fix a $\gamma$ value that was independent of temperature for each of the microstructures. This was done by first fitting the data for a temperature range in which the whole $\alpha$ peak lies well within the experimental window and allowing $\gamma$ to float. The resulting $\gamma$ values were then averaged and the data were refit to obtain the value $\alpha$ for the HN function. The deviations in the fits were still within the limits of the experimental uncertainty (see the example in Fig. 4). The $\gamma$ values for each microstructure are listed in Table II. The variations in $\alpha$ as a function of temperature are presented and discussed subsequently.

\section{B. Time-temperature superposition}

In order to address the validity of time-temperature superposition we first carried out a model-free comparison of data sets at different temperatures. Figures 5-8 show plots of the loss part of $\epsilon^{\prime \prime}(\omega)$ normalized to the maximum position of the $\alpha$ relaxation peak on the frequency axis and its height. This scaling is equivalent to applying both a horizontal and a

TABLE II. Values of the Havriliak-Negami parameter $\gamma$ for each of the polybutadiene microstructures (see the text for a discussion).

\begin{tabular}{cc}
\hline \hline Sample & $\gamma$ \\
\hline PB7 & 0.32 \\
PB53 & 0.46 \\
PB70 & 0.48 \\
PB95 & 0.41 \\
\hline \hline
\end{tabular}




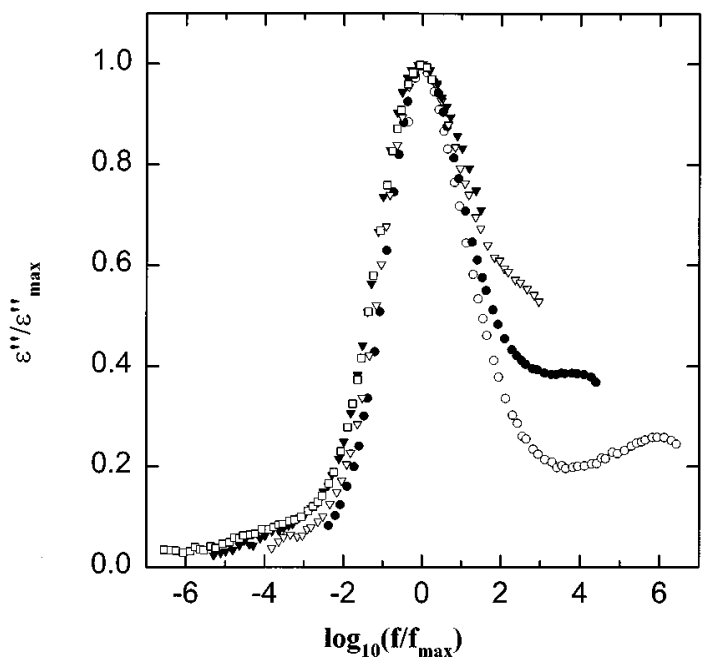

FIG. 5. Plot of the loss part of the dielectric function scaled to its maximum value, $\epsilon^{\prime \prime}(f) / \epsilon_{\max }^{\prime \prime}$, vs the logarithm of the frequency divided by the position of the maximum on the frequency axis, $f / f_{\max }$ for the sample PB 7. Temperatures: $(\bigcirc)-99.7^{\circ} \mathrm{C},(\bullet)-95.7^{\circ} \mathrm{C},(\nabla)-91.8^{\circ} \mathrm{C},(\nabla)-87.0^{\circ} \mathrm{C}$, ( $\square)-79.9^{\circ} \mathrm{C}$

vertical shift factor in logarithmic plots as is often done for rheological data. ${ }^{22}$ If the time-temperature superposition principle were valid, curves at different temperatures would superimpose onto a single master curve.

Figures 5-8 show that the scaling is poor for PB 7 (Fig. 5) while it is much better for all other microstructures (Figs. 6-8) for which only the lowest temperature data deviate from the master curve in some cases. Interestingly, the deviations in Fig. 5 seem to consist of a broadening of the peak with increasing temperature. This stands in contrast to the usual finding of the opposite temperature dependence in dielectric experiments, e.g., Refs. 21 and 23. Only in a few

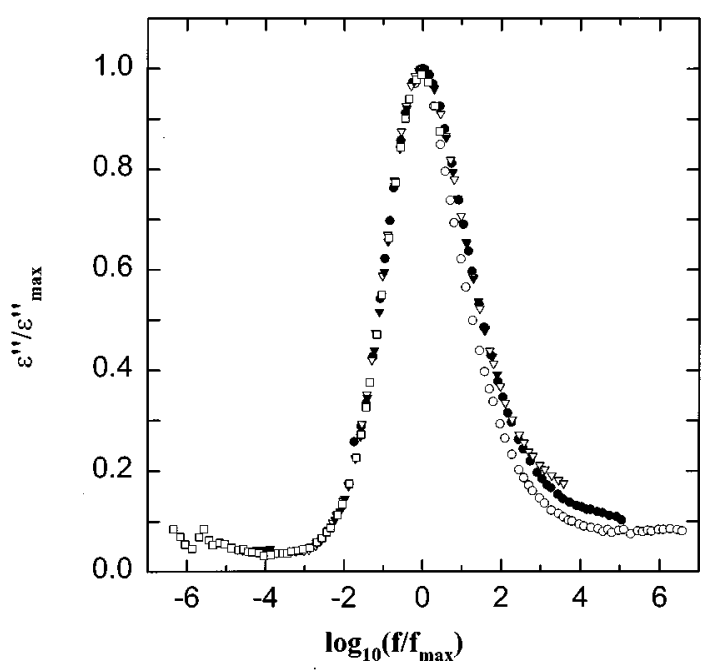

FIG. 6. Plot of the loss part of the dielectric function scaled to its maximum value, $\epsilon^{\prime \prime}(f) / \epsilon_{\max }^{\prime \prime}$, vs the logarithm of the frequency divided by the position of the maximum on the frequency axis, $f / f_{\max }$ for the sample PB 53. Temperatures: $(\bigcirc)-61.1{ }^{\circ} \mathrm{C},(\bullet)-57.0^{\circ} \mathrm{C},(\nabla)-52.6{ }^{\circ} \mathrm{C},(\boldsymbol{\nabla})-45.0^{\circ} \mathrm{C}$, ( $\square)-35.2{ }^{\circ} \mathrm{C}$

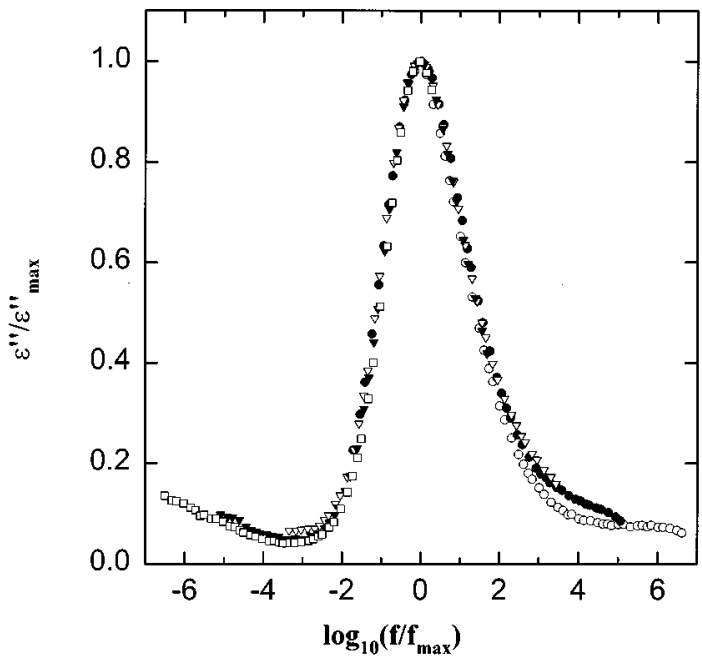

FIG. 7. Plot of the loss part of the dielectric function scaled to its maximum value, $\epsilon^{\prime \prime}(f) / \epsilon_{\max }^{\prime \prime}$, vs the logarithm of the frequency divided by the position of the maximum on the frequency axis, $f / f_{\max }$ for the sample PB 70. Temperatures: $(\bigcirc)-42.1{ }^{\circ} \mathrm{C},(-)-38.1{ }^{\circ} \mathrm{C},(\nabla)-33.2{ }^{\circ} \mathrm{C},(\nabla)-25.2{ }^{\circ} \mathrm{C}$, $(\square)-15.2{ }^{\circ} \mathrm{C}$.

cases (e.g., $\mathrm{Ca}_{0.4} \mathrm{~K}_{0.6}\left(\mathrm{NO}_{3}\right)_{1.4}$, Ref. 24) have dielectric spectra been reported which broaden with increasing temperature as in the case of the PB 7 material.

From the same figures (5-8) it can be seen that the $\beta$ relaxation (in relation to the $\alpha$ peak) is exceptionally strong in the case of PB 7. For all other microstructures the $\beta$ peak is considerably weaker. This result agrees with the findings in Ref. 18 for the same material.

The proximity to the $\alpha$ relaxation and the strength of the $\beta$ relaxation may also be reasons for the deviations from time-temperature superposition in the PB 7 data. On the other hand, the fact that for PB 7 the deviation from scaling

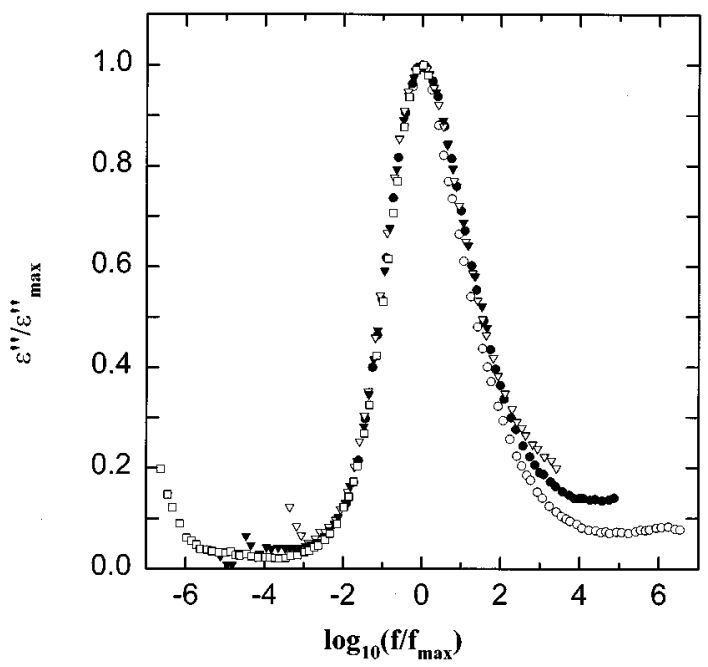

FIG. 8. Plot of the loss part of the dielectric function scaled to its maximum value, $\epsilon^{\prime \prime}(f) / \epsilon_{\max }^{\prime \prime}$, vs the logarithm of the frequency divided by the position of the maximum on the frequency axis, $f / f_{\max }$ for the sample PB 95. Temperatures: $(\bigcirc)-3.3{ }^{\circ} \mathrm{C},(\bullet)+0.7^{\circ} \mathrm{C},(\nabla)+4.7^{\circ} \mathrm{C},(\nabla)+12.1{ }^{\circ} \mathrm{C},(\square)$ $+22.1^{\circ} \mathrm{C}$. 


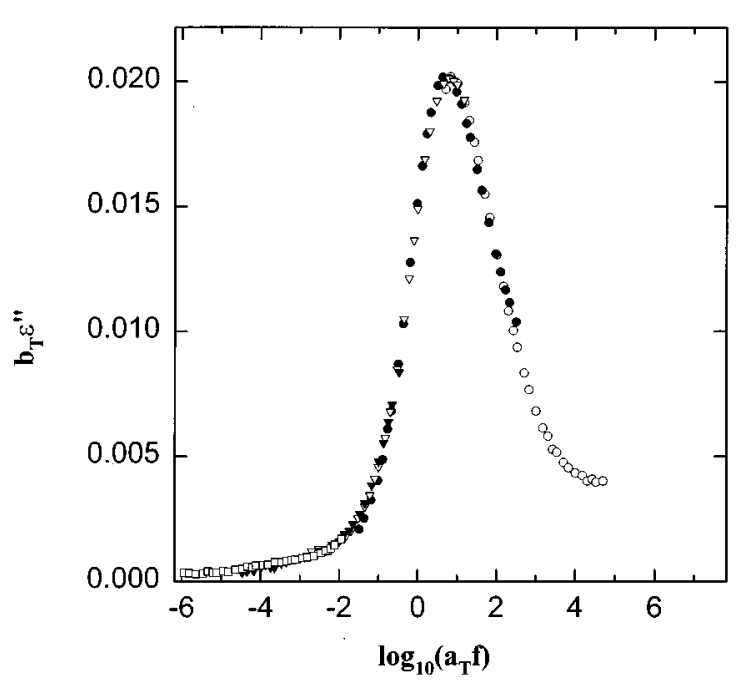

FIG. 9. Data as in Fig. 5 but with a frequency range restricted to $\omega$ $=0.01-100 \mathrm{rad} / \mathrm{s}$ and scaled by shift factors $a_{T}$ and $b_{T}$.

implies a change of slope of the low frequency wing of the $\alpha$ peak as well as of the high frequency wing indicates that it may not be simply a consequence of overlap with the $\beta$ relaxation. The question of whether or not the deviation from time-temperature superposition is an intrinsic feature of the $\alpha$ relaxation can only be answered fully if a valid model for the decomposition of the observed dielectric response into the $\alpha$ and $\beta$ contributions is used.

Another question as regards time-temperature superposition arises from the fact that the previous rheological investigations ${ }^{10}$ on the same sample did not show any indication of deviations from time-temperature superposition in the glassy dispersion region. ${ }^{25}$ An insight into the apparent differences in the superposability of the mechanical data and the dielectric data is obtained by considering Fig. 9, where the data of Fig. 5 are shown but for a frequency range that has been reduced to cover only the range on which the previous dynamic mechanical measurements were made, i.e., $\omega=0.01-100 \mathrm{rad} / \mathrm{s}$. The curves were then shifted by arbitrary horizontal and vertical shift factors $a_{T}$ and $b_{T}$ in order to obtain the best overlap. It can be seen that this restriction of the dynamical range leads to the impression that timetemperature superposition is possible. This result suggests that evidence for time-temperature superposition of the glassy dispersion in the mechanical measurements may require a greater time or frequency range than that normally covered in mechanical experiments.

In order to further examine the deviation from timetemperature superposition as an intrinsic feature of the $\alpha$ relaxation rather than simply the result of the overlapping $\alpha$ and $\beta$ relaxations, the exponent parameter $\alpha$ of the HN function in the fit described in the preceding section is shown in Fig. 10. Because the other exponent parameter, $\gamma$, was held constant for each of the microstructures, $\alpha$ is monotonically related to the "spectral width" - a smaller value of $\alpha$ indicating a broader glassy dispersion or $\alpha$ peak. It can be seen that only in the case of PB 7 is a strong decrease of this

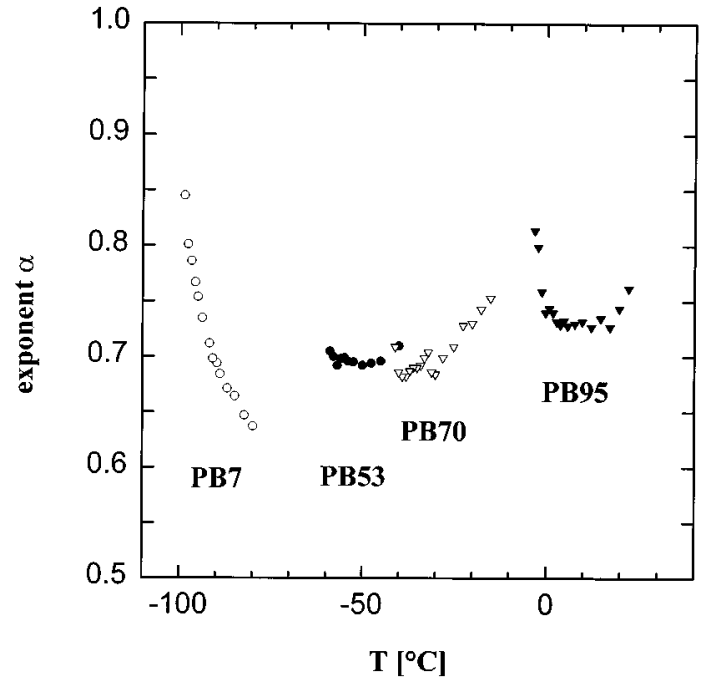

FIG. 10. Exponent parameter $\alpha$ in the Havriliak-Negami function representing the $\alpha$ relaxation in the fits described in Sec. III for all microstructures. Only those temperatures have been included where the $\alpha$ maximum was contained in the experimental frequency window.

parameter with temperature evident. For all other microstructures the variation of $\alpha$ is smaller and takes place mainly at the limits of the accessible temperature range. There, the precision of the fits may already be degraded because the wings of the HN function extend far outside the experimentally accessed frequency range.

In summary, we conclude that the evidence for a deviation from time-temperature superposition in the $\alpha$ relaxation of PB7 is not compelling because the direct comparison of the data is affected by the overlap with the $\beta$ relaxation while any model fit may be doubted because it is based on empirical functions. Nevertheless, we think that it is likely that such a deviation exists because both treatments suggest the same temperature dependence, i.e., a broadening of the spectrum of the $\alpha$ relaxation as temperature increases. We also note again that this broadening with increasing temperature is unusual.

\section{A data analysis issue}

Another issue in dealing with the experimental data arises when one compares two different methods of data analysis for essentially the same data. Hofmann et al. ${ }^{18}$ have investigated the same polybutadiene samples by frequencydomain dielectric spectroscopy on a slightly higher frequency range $\left(10^{-2}-10^{6} \mathrm{~Hz}\right)$. At first glance, the exponent parameters obtained here seem irreconcilable with those reported by them, especially in the case of PB 7: For $T$ $=-83.2{ }^{\circ} \mathrm{C}$ a value of $\beta=0.42 \pm 0.02$ is reported for the exponent parameter of the KWW expression (4.2). The latter was obtained by the approximate relation ${ }^{26,27}$ between $\mathrm{KWW}$ and $\mathrm{HN}$ parameters

$$
\beta=(\alpha \gamma)^{0.813} \text {. }
$$


If we apply the same equation to our values of $\alpha$ and $\gamma$ at $T=-83.2^{\circ} C$, we obtain $\beta=0.28 \pm 0.01$. The reason for this discrepancy is that we used $\gamma=$ const $=0.32$ as a restraint in the HN fit, while

$$
\gamma=1-0.812(1-\alpha)^{0.387}
$$

was used in Ref. 18 in order to restrain the parameters of the $\mathrm{HN}$ function to those which make the function itself an approximation of the Fourier transform of the KWW function. When we repeated some of the fits of our data with restraint (4.6) we obtained similar values of $\beta$ as reported in Ref. 18 and fits similar to those shown in Fig. 3 therein.

This may be regarded as a signal that it may be dangerous to overinterpret the significance of exponent parameters from fitting the HN, KWW, or similarly "flexible" fitting functions. The parameters may depend strongly on details of the fitting procedure. This complicates comparisons of published values from different sources as well as the physical interpretation of the parameters themselves.

\section{Temperature dependence of dielectric and mechanical measurements}

Because the dielectric data reported here and the rheological data obtained previously ${ }^{10}$ are for the same polybutadiene samples and were measured in the same laboratory, the $\alpha$ process measured from both methods can be compared. An elementary question is whether the temperature dependence of the dielectric and mechanical time scales is the same. For a comparison on an absolute time scale it is important how a characteristic time is defined, e.g., as the average relaxation time $\langle\tau\rangle$, as the inverse of the loss peak frequency $1 / \omega_{\max }$, or by using characteristic times from fitting functions such as $\tau_{\mathrm{HN}}$ or $\tau_{\mathrm{KWW}}$. Only in the case of a simple Debye relaxation do all values coincide. For a relative comparison this question is of lesser importance because all characteristic times are proportional if time-temperature superposition is applicable. Although in the case of the PB 7 this was not the case, the shape changes with temperature are not so dramatic that they invalidate the definition of a characteristic time completely.

For reasons of simplicity we chose to compare the inverse of the loss peak frequency $1 / \omega_{\max }$ of the dielectric function, $\epsilon^{\prime \prime}(\omega)$, with that of the dynamic modulus $G^{\prime \prime}(\omega)$ (Fig. 11) as a function of reciprocal temperature. In the case of the dielectric measurements the peak maximum was calculated from the $\mathrm{HN}$ parameters in order to eliminate the influence of the $\beta$ relaxation contribution and enable an extrapolation for temperatures at which the peak does not have its maximum within the frequency range of the experiment. For the rheological measurements the peak position was obtained from low temperature measurements in the glassy zone and then rescaled by the shift factor determined from a master curve construction. It has to be conceded thatespecially because of the difference of the shift factors in the glassy and terminal zones-this may already lead to significant errors when $T>T_{g}+10 \mathrm{~K}$. But because no rheological data are available for high frequencies there is no other way

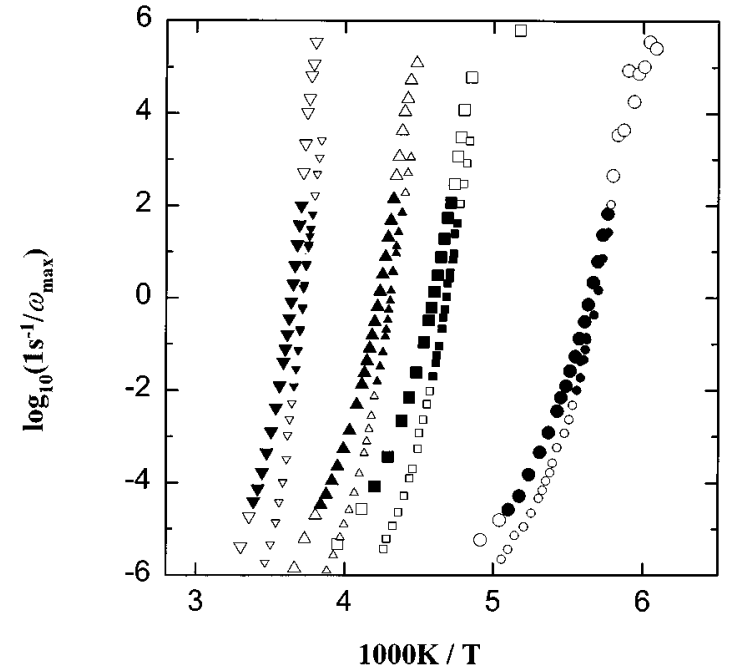

FIG. 11. Relaxation map for the characteristic times of dielectric (large symbols) and rheological (small symbols) measurements: the logarithm of the inverse maximum position $\omega_{\max }$ is plotted vs $1000 \mathrm{~K} / \mathrm{T}$. Samples: (O)

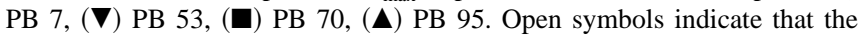
values are calculated by extrapolation, filled symbols that the maxima were actually present within the frequency range of the respective method.

to make a comparison in the high temperature regions. ${ }^{28}$ In order to indicate these sources of uncertainty, open symbols are used in Fig. 11 if the loss peaks are not within the dynamical range of the measurements. Those values were derived by extrapolations and therefore have to be considered with caution.

It can be seen immediately that the absolute values of the characteristic times are different for all microstructures. This is by no means surprising because there is no reason why the arbitrarily chosen characteristic time $1 / \omega_{\max }$ should be identical for dielectric and rheological measurements. A more interesting question is whether there are different temperature dependencies, in other words, whether or not dielectric and rheological characteristic times are proportional over the whole temperature range. Because this is difficult to judge from a relaxation map (Fig. 11), we plot the logarithm of the ratio, $\log _{10}\left(\omega_{\text {max,rheo }} / \omega_{\text {max,diel }}\right)$, in Fig. 12 . This value is conceptually similar to the "decoupling index" which was introduced by Angell ${ }^{29}$ and other authors ${ }^{24,30}$ to quantify differences in the temperature dependence of conductivity and mechanical relaxation.

It can be seen that for all microstructures but PB 95 the values do not indicate major differences in the temperature dependence. In this respect it is important to note that (1) the differences have to be viewed on a total dynamical range of 12 decades; (2) small errors in the absolute calibration of the temperature measurements may induce errors which are much larger than the apparent differences. The only case where significant differences occur is PB 95. There, a $3 \mathrm{~K}$ offset on the temperature scale would be necessary to explain the difference by experimental errors. Nevertheless, we do not want to make a strong point of this because in this case only four points could be calculated from maxima present within the dynamical range of dielectric and rheological 


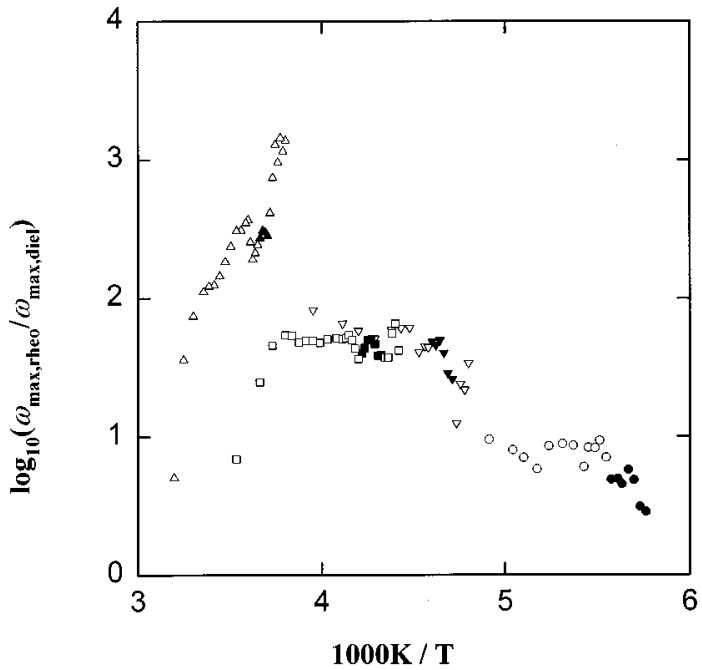

FIG. 12. The "decoupling index" $\log _{10}\left(\omega_{\text {max,rheo }} / \omega_{\text {max,diel }}\right)$. Open symbols indicate that the values are calculated by extrapolation in either the dielectric or the rheological data.

measurements and for the high temperatures the systematic error due to the difference of terminal and glassy shift factors is larger than for all other microstructures as pointed out in Ref. 1. This again calls for rheological experiments in the high frequency region which can match the frequency range of dielectric experiments.

There is only one fact which can clearly be stated for the decoupling index because it can be seen from the reliable ranges in Fig. 12 where no extrapolations were done (closed symbols): There is a tendency for the index to increase with increasing vinyl content. This means that the dielectric relaxation-in comparison to the rheological one-becomes slower if the polymer has more vinyl units. This finding is consistent with expectations from the DiMarzio-Bishop model of the relationship between dielectric and mechanical results. This is elaborated in Sec. IV E.

\section{E. Relationship between dielectric and mechanical relaxations: The DiMarzio-Bishop model}

In order to proceed beyond a simple comparison of the temperature dependencies of the characteristic times in rheological and dielectric measurements one has to assume a microscopic model for the connection between the measured quantities, e.g., the complex dynamic modulus $G^{*}(\omega)$ and the complex dielectric function $\epsilon^{*}(\omega)$. Several such models were compared in a recent article. ${ }^{31}$ From these we chose the model of DiMarzio and Bishop (DB) $)^{32}$ because it allows us to use the data which are readily available from this study and the previous rheological measurements. ${ }^{10,33}$

The result of the DB model is a very simple relation:

$$
\frac{\epsilon^{*}(\omega)-\epsilon_{\infty}}{\epsilon_{0}-\epsilon_{\infty}}=\frac{1}{1+K G^{*}(\omega)},
$$

which can formally be obtained by replacing the viscosity in the Debye model ${ }^{36}$ by the dynamic viscosity $\eta^{*}$ $=-G^{*}(\omega) / i \omega$. [We note that the rigorous derivation of this
TABLE III. Parameters from the fits of the dielectric loss $\epsilon^{\prime \prime}(\omega)$ calculated by the DB relation (4.7) to the experimental values. The last column contains the effecting radii calculated by Eq. (4.8) imposing $\left(\epsilon_{0}+2\right) /\left(\epsilon_{\infty}+2\right)$ $=1$.

\begin{tabular}{cccccc}
\hline \hline Sample & $T_{\text {rheo }} /{ }^{\circ} \mathrm{C}$ & $T_{\text {diel }} /{ }^{\circ} \mathrm{C}$ & $\Delta \epsilon$ & $K\left(\times 10^{-9} \mathrm{~Pa}^{-1}\right)$ & $R(\mathrm{~nm})$ \\
\hline PB7 & -95.4 & -95.7 & 0.116 & 4.7 & 1.0 \\
PB7 & -91.6 & -91.8 & 0.087 & 10.1 & 1.3 \\
PB95 & -1.8 & -1.3 & 0.090 & 24.2 & 1.9 \\
PB95 & +3.5 & +3.7 & 0.083 & 23.8 & 1.9 \\
\hline \hline
\end{tabular}

relation $^{32}$ is much more difficult than the simplicity of Eq. (4.7) may suggest.] The constant $K$ is, in the case of spherical particles with a dipole moment, related to the particle radius by

$$
K=\frac{4 \pi R^{3}}{k T} \frac{\epsilon_{0}+2}{\epsilon_{\infty}+2} .
$$

Because the real situation is much more complexespecially in polymers $-K$ has to be treated as a fitting parameter and $R$ is the radius of an "effective sphere" replacing the dipolar unit.

The values $\epsilon_{0}$ and $\epsilon_{\infty}$ can, in principle, be obtained from the low and high frequency limits, respectively, of $\epsilon^{\prime}(\omega)$. In most of our measurements the limits were not attained. An additional complication arises for PB 7 because of its strong $\beta$ peak: It is highly questionable whether the $\beta$ relaxation can be treated exactly the same way as the $\alpha$ relaxation. Therefore, relation (4.7) may be valid only for the $\alpha$ relaxation contribution to $\epsilon^{*}$. Because of these uncertainties we treated $\epsilon_{0}-\epsilon_{\infty}$ as an additional fitting parameter and scrutinized only the imaginary part of relation (4.7):

$$
\epsilon^{\prime \prime}(\omega)=\left(\epsilon_{0}-\epsilon_{\infty}\right) \frac{K G^{\prime \prime}(\omega)}{\left[1+K G^{\prime}(\omega)\right]^{2}+\left[K G^{\prime \prime}(\omega)\right]^{2}},
$$

which contains only $\epsilon_{0}-\epsilon_{\infty}=\Delta \epsilon$ as a scaling factor.

Table III shows some representative fitting parameters from the fits to Eq. (4.9) and Figs. 13-16 exhibit the respective comparisons of $\epsilon^{\prime \prime}(\omega)$ from the DB relation and the actual data. It is important to note that, in addition to the small temperature differences between the nominal temperatures of the dielectric and mechanical measurements, there may be a temperature difference of up to $1{ }^{\circ} \mathrm{C}$ because of the uncertainty of the absolute temperature determinations. However, this would result in a shift of at most half of a decade on the frequency axis and should not affect the peak shape seriously because the deviations from timetemperature superposition are evident only over a larger temperature range. Hence these are not the cause of the lack of agreement between the model calculations and the experiment.

It can be seen immediately that the calculated dielectric loss part $\epsilon^{\prime \prime}(\omega)$ does not fit well to the experimental values. The calculated peaks are generally too narrow. Figure 13 shows the influence of a (forced) change of the parameter $K$. If $K$ is increased, the calculated curves become even narrower and $\Delta \epsilon$ changes to a smaller value to adjust the peak 


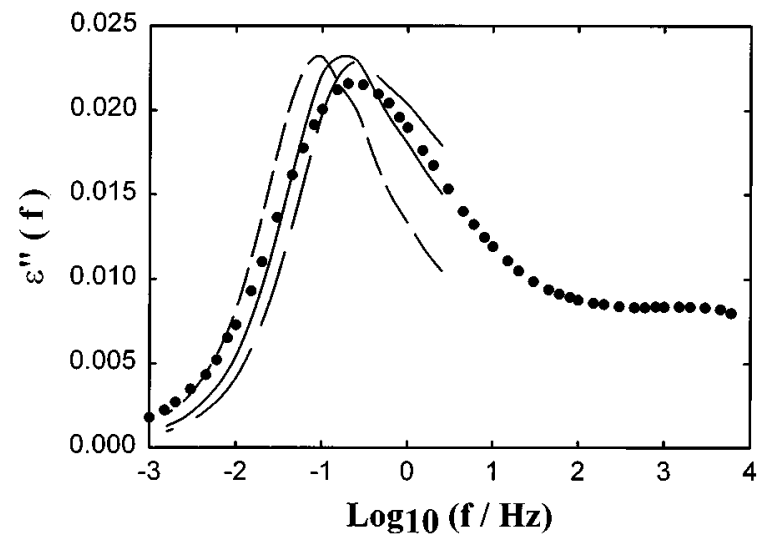

FIG. 13. Test of the DB model. Points: Loss part of the dielectric function $\epsilon^{\prime \prime}(\omega)$ of PB 7 at $-95.7^{\circ} \mathrm{C}$. Lines: $\epsilon^{\prime \prime}(\omega)$ calculated from the rheological dynamic moduli of PB 7 at $-95.4{ }^{\circ} \mathrm{C}$ using the DB model with the following parameter sets $K, \Delta \epsilon$ : (solid line, best fit; $4.7 \times 10^{-9} \mathrm{~Pa}^{-1}, 0.116$ ); (long dashed line, $2.5 \times 10^{-9} \mathrm{~Pa}^{-1}, 0.164$ ); (medium dashed line, $1 \times 10^{-8} \mathrm{~Pa}^{-1}$, 0.086 ).

height. If $K$ is decreased, the loss peak broadens and shifts to higher frequency, therefore the agreement is not improved by changing $K$.

In the case of PB 7 the overlap between $\alpha$ and $\beta$ relaxations may be invoked as an argument why the DB relation fails. If this were true, the fit should be better for PB 95 . Figures 15 and 16 show that the opposite is true. The discrepancy in the width between calculated and measured loss peaks seems to be even larger for PB 95 than for PB 7.

Despite the insufficiency of the DB fits themselves, the fitting parameters have reasonable values. The values of the effective sphere radii $R$ can be obtained by relation (4.8). In this calculation, the factor $\left(\epsilon_{0}+2\right) /\left(\epsilon_{\infty}+2\right)$ can be neglected because it is close to unity. The results shown in the last column of Table III lie in the range which would be expected for segmental reorientations. The temperature dependence of $R$ is small but there is a significant dependence on microstructure which can simply be explained by the fact that in

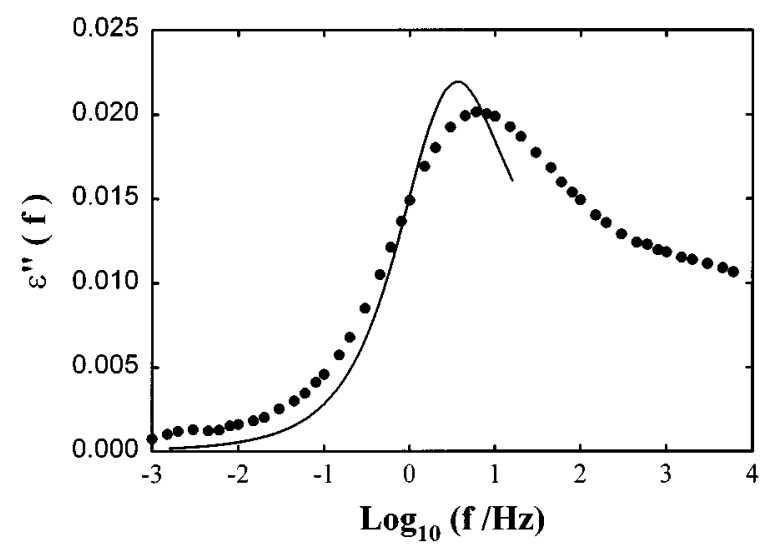

FIG. 14. Test of the DB model. Points: Loss part of the dielectric function $\epsilon^{\prime \prime}(\omega)$ of PB 7 at $-91.8^{\circ} \mathrm{C}$. Lines: $\epsilon^{\prime \prime}(\omega)$ calculated from the rheological dynamic moduli of PB 7 at $-91.6^{\circ} \mathrm{C}$ using the DB model with the parameters $K=10.1 \times 10^{-9} \mathrm{~Pa}^{-1}$ and $\Delta \epsilon=0.087$.

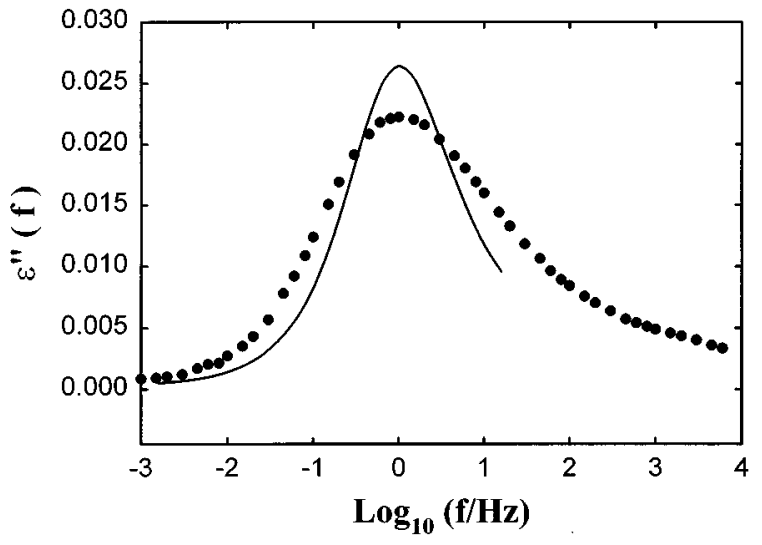

FIG. 15. Test of the DB model. Points: Loss part of the dielectric function $\epsilon^{\prime \prime}(\omega)$ of PB 95 at $-1.3^{\circ} \mathrm{C}$. Lines: $\epsilon^{\prime \prime}(\omega)$ calculated from the rheological dynamic moduli of PB 95 at $-1.8{ }^{\circ} \mathrm{C}$ using the DB model with the parameters $K=24.2 \times 10^{-9} \mathrm{~Pa}^{-1}$ and $\Delta \epsilon=0.090$.

PB 7 the dipole moment arises from cis monomeric units but from vinyl units for PB 95. It is clear that motions of the vinyl groups sweep out a larger volume than do those of the cis units and, therefore, should have a higher effective sphere radius. The values of $\Delta \epsilon$ are similar to those obtained from the HN fits, which lie in the range 0.103-0.110.

We note that an increase in $R$ implies an increase in $K$, which shifts the maximum of $\epsilon^{\prime \prime}(\omega)$ calculated by the DB model to lower frequencies. Therefore, the increase of $K$ with vinyl content is consistent with the increase of the ratio of dielectric and rheological characteristic times found above.

\section{SUMMARY AND CONCLUSIONS}

The availability of dielectric and rheological ${ }^{10}$ data from the same samples and measured in the same laboratory offers a unique opportunity to directly compare the electric and mechanical relaxation due to the $\alpha$ process. Here we report on measurements from a series of 1,2-1,4-polybutadienes

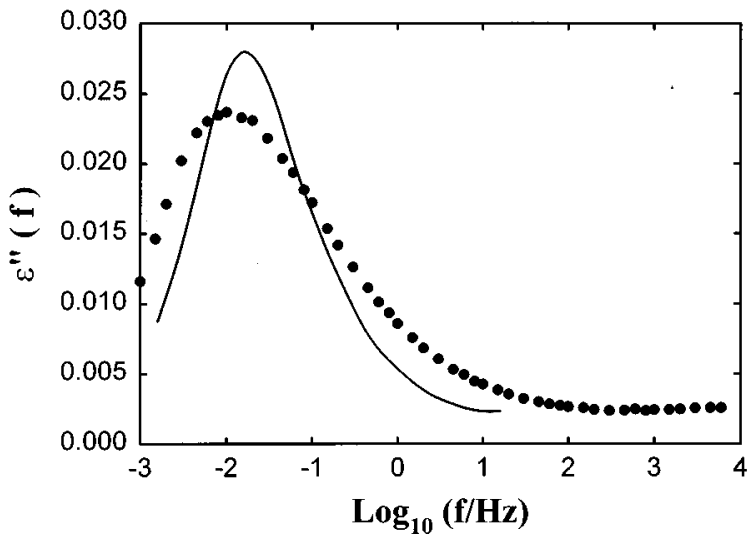

FIG. 16. Test of the DB model. Points: Loss part of the dielectric function $\epsilon^{\prime \prime}(\omega)$ of PB 95 at $+3.7^{\circ} \mathrm{C}$. Lines: $\epsilon^{\prime \prime}(\omega)$ calculated from the rheological dynamic moduli of PB 95 at $+3.5^{\circ} \mathrm{C}$ using the DB model with the the parameters $K=23.8 \times 10^{-9} \mathrm{~Pa}^{-1}$ and $\Delta \epsilon=0.083$. 
with a vinyl $(1,2)$ fraction ranging from 0.07 to 0.95 . Timedomain dielectric spectroscopy was used here to obtain the dielectric response over a large dynamic range and compare it with the mechanical response. Additionally, there data were used to reexamine the question of time-temperature superposition.

The temperature dependencies of the characteristic times were found to be identical for both dielectric and mechanical responses within the experimental uncertainties of the measurements. These arise mainly from the fact that the rheological measurements were restricted to a frequency range in which the glassy relaxation can only be observed for the lower temperatures near the glass transition. The extension of the temperature range by means of time-temperature superposition suffers from the well-known fact ${ }^{37}$ that the shift factors for different modes of motion (e.g., in the glassy and terminal zone) may differ. Therefore, the results presented here demonstrate how important it is that the dynamic range of rheological experiments be extended toward higher frequencies in order to fully scrutinize the possible identity of the temperature dependencies of dielectric and mechanical relaxations. The limitations of normal measurements to 4 decades in frequency or time (and even the extension to 6.7 decades) are obvious from the comparisons made here and are strong arguments for such measurements to be extended to 7-9 decades at a minimum. Similarly, dielectric experiments would be significantly enhanced by extension to a larger frequency range.

Nevertheless, in the case of our data, a temperature range where both methods give reliable results could be found for each of the microstructures. A comparison with regard to the vinyl content yields an increase of the dielectric characteristic times with respect to the rheological ones if the vinyl content is increased. This result is consistent with the qualitative expectation from the DiMarzio-Bishop (DB) ${ }^{32}$ model. According to this model the higher volume swept out by the vinyl groups (i.e., the effective sphere radius in the model) as compared to the cis groups leads to an increase in the parameter $K$ in Eq. (4.7), which relates the complex dynamic modulus and the complex dielectric function. This, in turn, shifts the dielectric loss peak toward lower frequencies.

On the other hand, the DB model was not confirmed quantitatively. The model [Eq. (4.7)] always predicts a narrower loss peak than is actually observed in the dielectric data. Several reasons for this were already given by the authors: ${ }^{32}$ (1) No distribution in the effective sphere radii was assumed. (2) Neither an electrical nor a mechanical coupling of the individual dipoles is included. Especially for polymers the latter coupling is important because the dipolar monomeric units are connected by covalent bonds. (3) No anisotropy of the dipolar units or their local environment was taken into account. All these factors would increase the width of the resulting dielectric loss peak if they could be implemented in the model. Unfortunately, the molecular details necessary for doing this exceed our current capabilities.

The larger dynamic range of the dielectric data made examination of time-temperature superposition behavior more solid than the rheological experiments ${ }^{10}$ permitted. Es- pecially for PB 7 (0.07 vinyl fraction) we show that the obvious deviations from time-temperature superposition which occur when one attempts to construct a master curve from the $\epsilon^{\prime \prime}(\omega)$ data vanish when one reduces the frequency range to that of the rheological experiments. This is again an argument for extending the dynamical range in rheology.

Nevertheless, direct comparison of the loss peak was still limited to a temperature range of about $25 \mathrm{~K}$ due to the frequency window of our dielectric apparatus (6.7 decades). Therefore, even in dielectric experiments the dynamical range should be improved beyond the 7 decades obtained here. In principle, this can be done by combining different dielectric techniques ${ }^{21,23,38}$ but currently some of these techniques do not have the resolution necessary to apply in the case of materials with a low dielectric response as, e.g., polybutadiene. However, we do note that for the polybutadiene microstructures with vinyl content $\geqslant 0.53$ we could not detect significant deviations from time-temperature superposition in either dielectric or mechanical measurements.

In the case of PB 7, where the dielectric data encompasssignificant portions of both $\alpha$ and $\beta$ relaxations, the question does remain open whether the apparent deviation from timetemperature superposition is due to the overlap of the $\alpha$ and $\beta$ relaxations (the latter being strongest for PB 7 among all microstructures) or an intrinsic feature of the $\alpha$ relaxation. The fit with a combination of Havriliak-Negami (HN) and Cole-Cole functions shows a significant change of the width of the $\mathrm{HN}$ function, i.e., the $\alpha$ contribution. This supports the view that the violation of the time-temperature superposition principle is an intrinsic property of the $\alpha$ relaxation. But model fits of this sort constitute no proof unless the models can be justified on microscopic grounds, i.e., by a theory of the motion of individual molecular dipoles. To our knowledge none of the model functions currently used (HN, KWW, etc.) fulfills this criterion and in addition the rules which have to be applied when different relaxations merge are still a subject of discussion. ${ }^{9}$

Furthermore, by comparing our results with those of a similar dielectric investigation, ${ }^{18}$ one recognizes that parameters derived from such models are dependent on subtle assumptions. Therefore, the comparison of results from different authors may be difficult.

\section{ACKNOWLEDGMENTS}

R.Z. has been supported by Deutsche Forschungsgemeinschaft and wishes to thank the National Institute of Standards and Technology for its support during his stay.

\footnotetext{
${ }^{1}$ N. G. McCrum, B. E. Read, and G. Williams, Anelastic and Dielectric Effects in Polymeric Solids (Wiley, London, 1967).

${ }^{2}$ S. Havriliak and S. Negami, J. Polym. Sci., Part C 14, 99 (1966).

${ }^{3}$ F. Kohlrausch, Poggendorff's Annalen der Physik 12, 393 (1847)

${ }^{4}$ G. Williams and D. C. Watts, Trans. Faraday Soc. 66, 80 (1970).

${ }^{5}$ R. H. Cole and K. S. Cole, J. Chem. Phys. 10, 98 (1942).

${ }^{6}$ L. Wu and S. R. Nagel, Phys. Rev. Lett. 46, 11198 (1992).

${ }^{7}$ G. P. Johari, J. Chem. Phys. 58, 1766 (1970).

${ }^{8}$ F. Garwe, A. Schönhals, M. Beiner, K. Schröter, and E. Donth, J. Phys., Condens. Matter 6, 6941 (1994).

${ }^{9}$ A. Arbe, D. Richter, J. Colmenero, and B. Farago, Phys. Rev. E 54, 3853 (1996)
} 
${ }^{10}$ R. Zorn, G. B. McKenna, L. Willner, and D. Richter, Macromolecules 28, 8552 (1995).

${ }^{11}$ C. A. Angell and W. Sichina, Ann. N.Y. Acad. Sci. 279, 53 (1967).

${ }^{12}$ C. A. Angell, J. Phys. Chem. Solids 49, 863 (1988).

${ }^{13}$ M. Morton and L. J. Fetters, Rubber Chem. Technol. 48, 359 (1975).

${ }^{14}$ F. I. Mopsik, Rev. Sci. Instrum. 55, 79 (1984).

${ }^{15}$ F. I. Mopsik, IEEE Trans. Electr. Insul. EI-20, 957 (1985).

${ }^{16}$ The absolute values of $\epsilon^{\prime \prime}$ obtained here for PB 7 are $\approx 26 \%$ higher than those published by Quan et al. (Ref. 17) for 11\% 1,2-polybutadiene at 1 $\mathrm{kHz}$ and $\approx 31 \%$ higher than those published by Hofmann et al. (Ref. 18) for a $7 \%$ 1,2-polybutadiene at $-92.1{ }^{\circ} \mathrm{C}$.

${ }^{17}$ X. Quan, G. E. Johnson, E. W. Anderson, and F. S. Bates, Macromolecules 22, 2451 (1989).

${ }^{18}$ A. Hofmann, A. Alegría, J. Colmenero, and L. Willner, Macromolecules 29, 129 (1996).

${ }^{19}$ F. I. Mopsik, in Electrically Based Microstructural Characterization, edited by R. A. Gerhard, S. A. Taylor, and E. J. Garboczi [Mater. Res. Soc. Symp. Proc. 147, 357 (1996)].

${ }^{20}$ R. D. Deegan and S. R. Nagel, Phys. Rev. B 52, 5653 (1995).

${ }^{21}$ P. K. Dixon, L. Wu, S. R. Nagel, B. D. Williams, and J. P. Carini, Phys. Rev. Lett. 65, 1108 (1990).

${ }^{22}$ J. D. Ferry, Viscoelastic Properties of Polymers (Wiley, New York, 1961).

${ }^{23}$ A. Schönhals, F. Kremer, and E. Schlosser, Phys. Rev. Lett. 67, 999 (1991).

${ }^{24}$ F. S. Howell, H. A. Bose, P. B. Macedo, and C. T. Moyniham, J. Chem. Phys. 78, 639 (1974).

${ }^{25}$ Deviations from thermorheological simplicity were only detected when glassy and terminal dispersion shift factors were compared. Each of the dispersions individually followed the time-temperature superposition principle.

${ }^{26}$ F. Alvarez, A. Alegría, and J. Colmenero, Phys. Rev. B 44, 7306 (1991).
${ }^{27}$ F. Alvarez, A. Algería, and J. Colmenero, Phys. Rev. B 47, 125 (1993).

${ }^{28}$ An estimate of the systematic error which results from doing this can be obtained by extrapolation of the mismatch between glassy and terminal shift factors in the overlap region reported in Ref. 10 to the highest temperature used here. For PB 7 through PB 70 we expect errors less than a factor of 2, i.e., 0.3 offset on the logarithmic scales of Figs. 11 and 12. Only in the case of PB 95 the factor may rise to about 15, i.e., an offset of 1.2 on the logarithmic scales, for the highest temperature because there the observed mismatch was much larger than for the other microstructures.

${ }^{29}$ C. A. Angell, Solid State Ion. 9\&10, 3 (1983).

${ }^{30}$ C. T. Moynihan, N. Balitactac, L. Boone, and T. A. Litovitz, J. Chem. Phys. 55, 3013 (1971).

${ }^{31}$ S. Havriliak, Jr. and S. J. Havriliak, J. Polym. Sci. B, Polym. Phys. 33, 2245 (1995).

${ }^{32}$ E. A. DiMarzio and M. Bishop, J. Chem. Phys. 60, 3802 (1974).

${ }^{33}$ Among the other models reported only the one of Havriliak and Havriliak (HH) (Refs. 34 and 35) seems to be sufficiently elaborate to accommodate the complicated situation of polymer viscoelasticity. The HH model needs the tensile creep compliance $D(t)$ as an input of rheological data. The conversion of the rheological data in Ref. 10 would require a transform into the time domain. Because of the complication and numerical uncertainty involved with this we did not test the HN model. Additionally, we point out that Havriliak and Havriliak (Ref. 31) showed that the results of their model and the DB model are very similar despite their different derivations.

${ }^{34}$ S. Havriliak, Jr., Macromolecules 23, 2384 (1990).

${ }^{35}$ S. Havriliak, Jr. and S. J. Havriliak, Polymer 33, 938 (1991).

${ }^{36}$ P. Debye, Polar Molecules (Dover, New York, 1929).

${ }^{37}$ K. L. Ngai and D. J. Plazek, Rubber Chem. Technol. 68, 376 (1995).

${ }^{38}$ C. Cramer, K. Funke, and T. Saatkamp, Philos. Mag. B 71, 701 (1995). 